\title{
THE CHALLENGE OF TIME-RESOLVED FLOW INVESTIGATION OF A ONE-STAGE CENTRIFUGAL COMPRESSOR WITH A NON-SYMMETRIC VOLUTE
}

\author{
M. Voges ${ }^{*}-J$. Klinner ${ }^{*}-$ C. Willert ${ }^{*}-$ A. Bassetti ${ }^{*}-$ O. Reutter $^{*}-$ M. van Rooij ${ }^{+}$ \\ *German Aerospace Center (DLR), Institute of Propulsion Technology, Cologne, Germany, \\ melanie.voges@dlr.de \\ ${ }^{+}$Royal Netherlands Aerospace Center (NLR), Dept. of Flight Physics and Loads, Amsterdam, \\ The Netherlands
}

\begin{abstract}
A single stage industrial centrifugal compressor including vaneless diffuser and a nonsymmetric volute is investigated with regard to the development of flow instabilities and rotating stall. A deeper understanding of flow phenomena that might be a precursor for stall inception would help to predict unstable operation already during the design phase of a compressor stage, as today the design is commonly supported by CFD analysis providing performance data and flow information at an early stage of the design process. Due to the complex flow path geometry of the volute a sophisticated instrumentation concept was developed allowing for unsteady pressure measurement with synchronized high-resolution flow field measurement. This paper summarizes the challenges and the steps taken during rig design in terms of integrating non-intrusive, high resolution pressure measurement sensors along with access for optical techniques such as time-resolved PIV and L2F to investigate unsteady flow phenomena and stall inception occurring at reduced speed operation. Results of performance measurements and surge testing are presented and compared to CFD simulations. Exemplary results of the extensive database obtained by optical techniques in combination with dynamic pressure measurements are provided.
\end{abstract}

KEYWORDS

rotating stall, radial compressor, time-resolved PIV

\begin{tabular}{ll}
\multicolumn{2}{c}{ NOMENCLATURE } \\
BPF & blade passing frequency \\
L2F & Laser-2-Focus technique \\
LE & leading edge \\
LES & large eddy simulation \\
LTS & Liebherr Aerospace Toulouse SAS \\
MPS & mean power spectra \\
TR-PIV & time-resolved particle image velocimetry \\
PS & pressure side \\
r & radius \\
RANS & Reynolds averaged Navier Stokes \\
RI & rotating instability \\
rpm & revolutions per minute \\
SS & suction side \\
TE & trailing edge \\
URANS & unsteady Reynolds averaged Navier Stokes
\end{tabular}




\section{INTRODUCTION}

Centrifugal compressor stages using vaneless diffusers are known to have a wide operating range. However, when operated outside of nominal performance, instabilities have been reported to commonly develop in the impeller (rotor) or diffuser or both (Sorokes, 2003). The unsteady flow phenomena are believed to start from a localized flow separation (stall) that frequently will orbit within the stage at sub-harmonic rotational speeds (rotating stall). If not damped sufficiently the stall cells will eventually develop into more hazardous self-excited pressure oscillations within the entire compressor stage leading to surge phenomena that can induce high aero-elastic loads on the impeller itself leading to premature aging (fatigue) or ultimate failure of the stage. Predicting the onset of the instabilities is a prerequisite to maintain compressor operating within safe operating margins. In practice this can be achieved through online pressure monitoring with active feedback (throttle valves) or through passive measures such as casing treatments or bleed slots (Fisher, 1988, Yamaguchi et al., 2002, Guillou et al., 2012)). Nonetheless the physical understanding and associated modelling of the stall and surge inception is rather limited and mostly restricted to cause-and-effect investigations. With improved computational models being available nowadays, in particular URANS and LES, more in-depth investigations of the underlying flow physics have become possible (see e.g. Dickmann et al., 2006, Bousquet, 2014) but rely on high quality experimental data for validation. Furthermore, the majority of the literature focuses on vaned diffuser stages (Trébinjac et al., 2011, Pullan et al. 2012 Bousquet, 2014). An effort would be necessary on vaneless diffuser cases, although the scientific output had a stronger focus on that in the last decade as detailed by Pavesi et al. (2011), Dazin et al. (2009) and Ferrara et al. (2004). Moreover, due to the various configurations described in the literature, a large spectrum of stall and surge behavior is reported and up to now, no overall rule can be given a priori concerning the instability inception in a given machine.

The aim of the present H2020 CleanSky2 research project ROSSINI is two-fold: on the one hand to determine a surge inception scenario for a given industrial centrifugal compressor including vaneless diffuser along with a non-symmetric volute at the outlet. Detailed numerical and experimental investigations are performed with the aim of improving the understanding of the complex flow phenomena leading to flow instabilities and surge. Based on the results obtained, the numerical models necessary to faithfully capture the unsteady flow features at near stall operation will be determined and validated. The second project aim is the analysis of the numerical and experimental results in order to establish a theoretical approach supporting the prediction of instabilities leading to rotating stall and surge. The ultimate (long-term) objective is to develop an analytical model to predict the critical operating conditions in compressors and associated rotating instabilities (RI) therein. The model could be based on a linear stability analysis of laminar 2D flow profiles to determine the unstable modes and the associated frequencies. Such a method would be applicable early in the design process of industrial turbomachinery components.

\section{TEST CASE}

The centrifugal compressor stage provided by the industrial partner Liebherr Aerospace Toulouse SAS (LTS) consists of an impeller with 15 backswept, unshrouded main blades without splitter blades, a vaneless diffuser and an asymmetric volute, as shown in Figure 1. In its original industrial application, the compressor stage is operated inside of the cabin air conditioning system of a civil aircraft. At the design point the rotational speed is about 48,000 rpm and the total pressure ratio of the stage is about 1.8. The inlet and outlet sections consist of straight pipes (not shown in Figure 1). Due to the volute flow path overlapping both, the diffuser section and partly the inlet section of the wheel (impeller), an assessment was performed using steady RANS calculations and 3D design tools to find the best suitable solution for rig instrumentation.

\section{METHODOLOGY}

Preliminary steady RANS calculations of the LTS centrifugal compressor stage were performed using the DLR TRACE code (for details see e.g. Weber et al. (2016)) in order to find a precursor of the expected stall inception or beginning flow separation for a defined number of operating conditions. 
In a following step, the stage dimensions were scaled in the CFD environment to match DLR's existing facility inlet pipe diameter and at the same time to ensure that stage performance would still be comparable to the original test case. Rotational speed of the rig has been scaled together with the geometry keeping Mach similarity. The ROSSINI compressor stage dimensions are summarized in Table 1. Details of the numerical setup have been presented by Rosemeier (2017).

a)

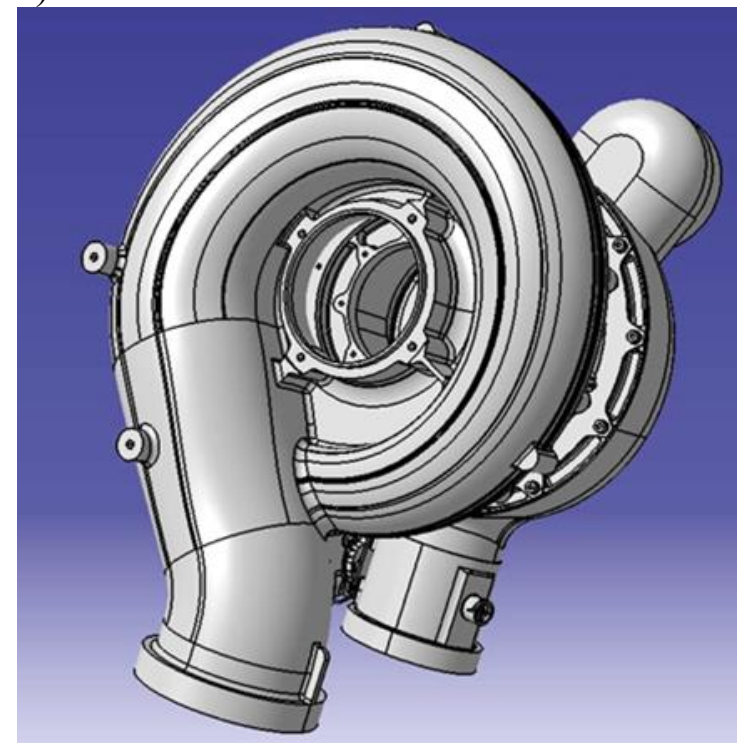

b)

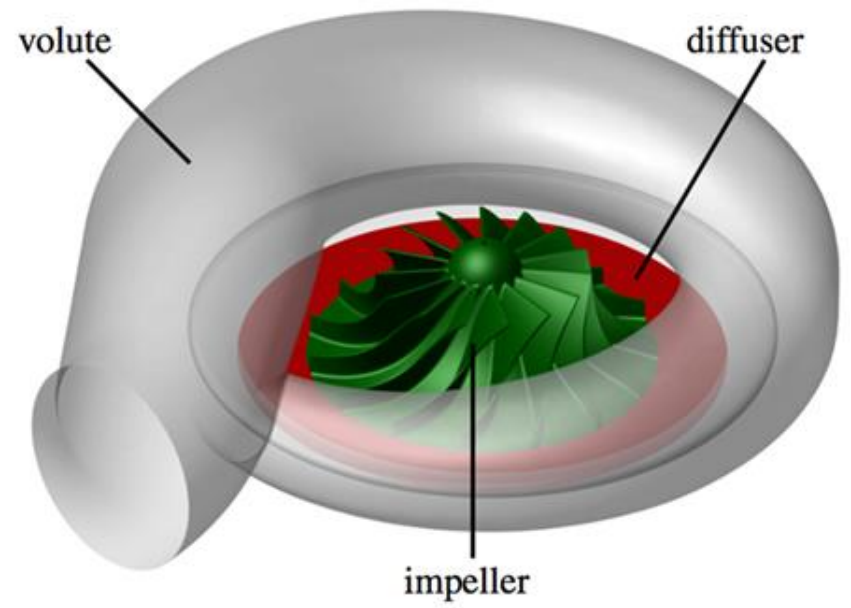

Figure 1 a) Industrial centrifugal compressor stage with vaneless diffuser and asymmetric volute; b) numerical model of the ROSSINI compressor stage (by [Rosemeier 2017])

Table 1 ROSSINI compressor stage dimensions

\begin{tabular}{|l|l|}
\hline Number of blades & 15 \\
\hline Shroud (inlet) radius $\mathrm{r}_{\mathrm{s}}$ & $79.37 \mathrm{~mm}$ \\
\hline Impeller LE tip radius $\mathrm{r}_{1}$ & $78.97 \mathrm{~mm}$ \\
\hline Impeller TE tip radius $\mathrm{r}_{2}$ & $127.84 \mathrm{~mm}$ \\
\hline Diffuser exit radius $\mathrm{r}_{3}$ & $214.13 \mathrm{~mm}$ \\
\hline Diffuser passage height $\mathrm{h}$ & $14.37 \mathrm{~mm}$ \\
\hline
\end{tabular}

The numerical investigation confirmed the existence of growing detached flow inside of the impeller blade passage close to the surge line (i.e. the numerical stability limit), that descent from tip clearance flow (indicated by a red arrow in Figure 2) and vortex development and lead to a growing blockage area in the blade passages with reduced mass flow. The therefore reduced velocities in the tip region and at the pressure side (PS) further downstream of the blades are a precursor for flow separation and possible beginning of stall inside the blade passage (see Mach number distributions in Figure 2).

The volute flow field is dominated by vortex structures in circumferential direction, mainly induced by the exit flow angle of the wheel and the overall geometry of the volute. Secondary vortex structures are formed due to the flow deflection at the exit of the diffuser when entering the almost circular cross-section of the volute, as indicated in Figure 3.a. 
a)

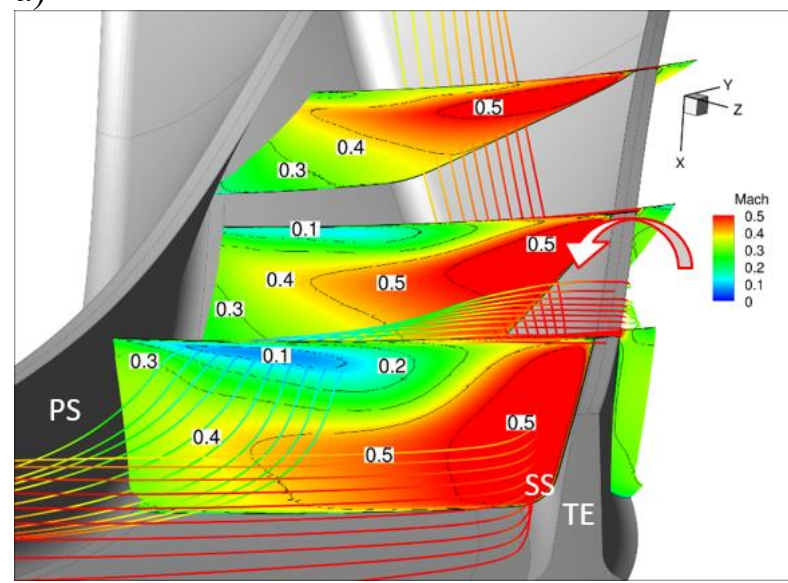

b)

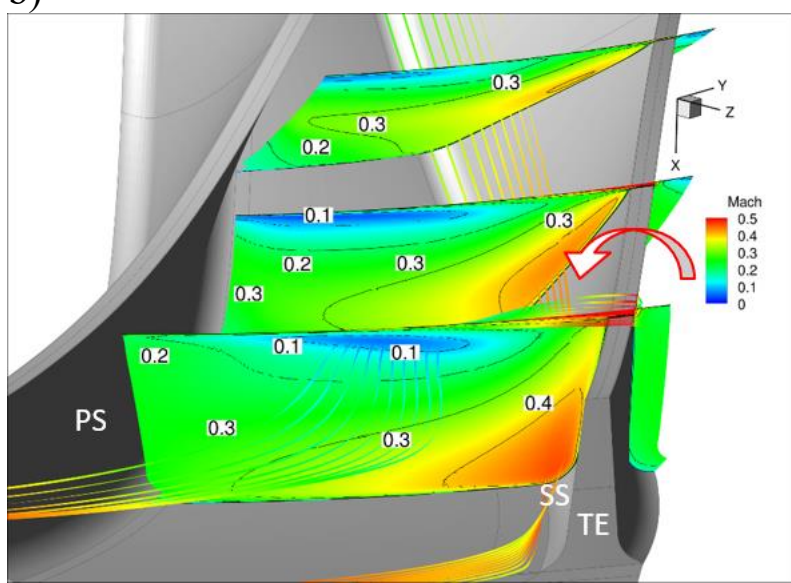

Figure 2: TRACE Mach number distribution for different S3-planes in the downstream part of a blade passage; a) peak efficiency and b) near stall operation, both at $100 \%$ speed.

The shape of the secondary vortex structure changes with mass flow and can consist of a single vortex filling the whole passage (close to choke) up to counter-rotating vortex systems at peak efficiency and near stall operation, as shown in Figure 3.b.

Special attention was paid to the "tongue" of the volute flow path where the smaller channel section discharges into the larger outlet section (marked with a blue arrow in Figure 3(a)). The steady RANS calculations revealed a pressure peak at the tongue in the outlet section (and related velocity peak on the adjacent side). In addition, a certain amount of flow is recycled into the smaller channel section (visible following the stream traces given in Figure 3.a near the blue arrow) and recirculates a second time with the main structures exiting the diffuser section.

a)

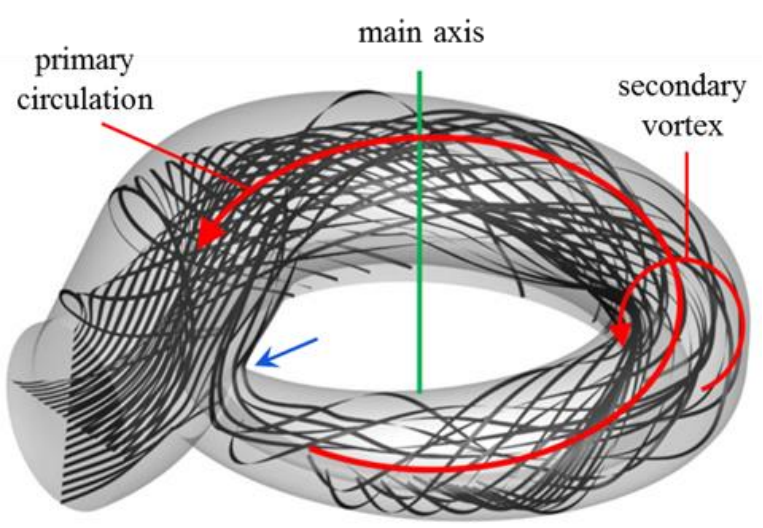

b)

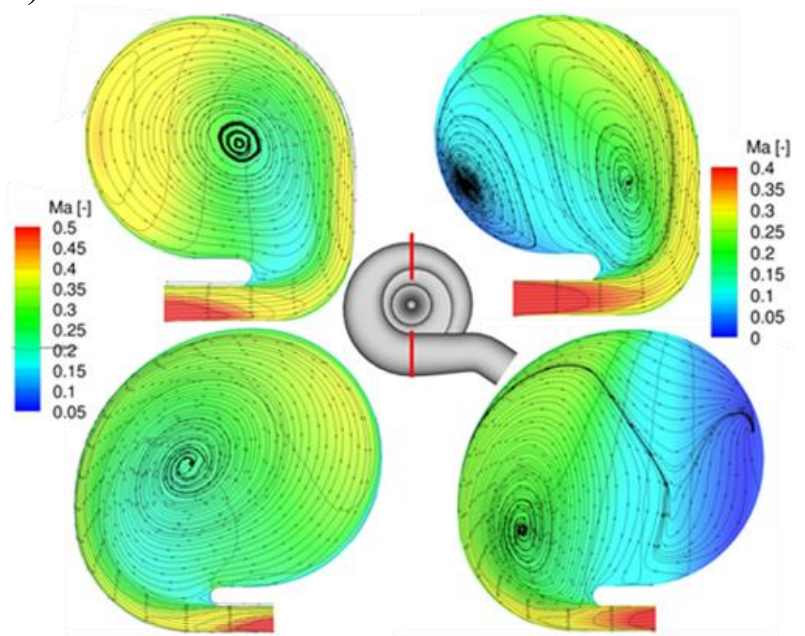

Figure 3: a) Vortex flow structures inside the volute; b) Vortex formation in the volute for different cross sections; left: peak efficiency, right: near stall operation (i.e. num. stability limit)

\section{DESIGN AND INSTRUMENTATION CONCEPT}

The focus for flow diagnostics and unsteady instrumentation was on the impeller inlet and outlet flow field as well as in the volute with emphasis on the tongue. For standard performance analysis the shaft speed, tip clearance, mass flow rate as well as total temperatures and pressures upstream and downstream of the stage were monitored. An appropriate number of static pressure transducers was needed along the shroud to analyze the pressure rise through the stage, impeller flow quality and diffuser or volute recovery. 


\section{Rig Design and Infrastructure}

With the chosen scaling factor, it was possible to keep the entire inlet section of the centrifugal compressor facility, including venturi type mass flow device, settling chamber, bell-mouth and inlet diameter of the impeller (identical to other DLR impellers, such as SRV5, see Elfert et al. (2017)). Maintaining the axial position of the impeller inlet plane on the shaft allowed for keeping bearings and using the same method for balancing of the wheel, which resulted in a comparable dynamic behavior of the shaft system.

A new approach was needed combining all diagnostic equipment in one single casing, allowing for simultaneous application of laser-based flow field measurements as well as static and dynamic pressure measurements and stage performance monitoring - at the same time the stage performance should not be influenced by the integrated measurement equipment. In the realized approach the entire casing was built in a split-design where the inner casing (lid) carries most of the instrumentation needed for performance monitoring and investigation of unsteady flow phenomena.

\section{Optical Diagnostic and Pressure Instrumentation}

The investigation of flow instabilities and unsteady phenomena leading to stall or surge is a challenging task in real turbomachinery component testing. The combined (simultaneous) application of conventional instrumentation (static temperature and pressure taps) with advanced, high resolution dynamic pressure sensors and the optical techniques high speed PIV and L2F requires specific procedures for mounting in terms of available space (cable routing, dimensions of sensor itself), measurement accuracy (precise adjustment, sealing, cleanliness) and of course direct access ("easy" revision, mounting and disassembly) before and after rig operation.

These aspects together with the asymmetric geometry of the present compressor casing resulted in the modular assembly structure of the stage and an instrumentation concept described in the following. For better orientation, a 3D view of the stage design is given in Figure 4(a), indicating the 8 static pressure taps along the outer circumference of the volute flow path (red arrows).

a)

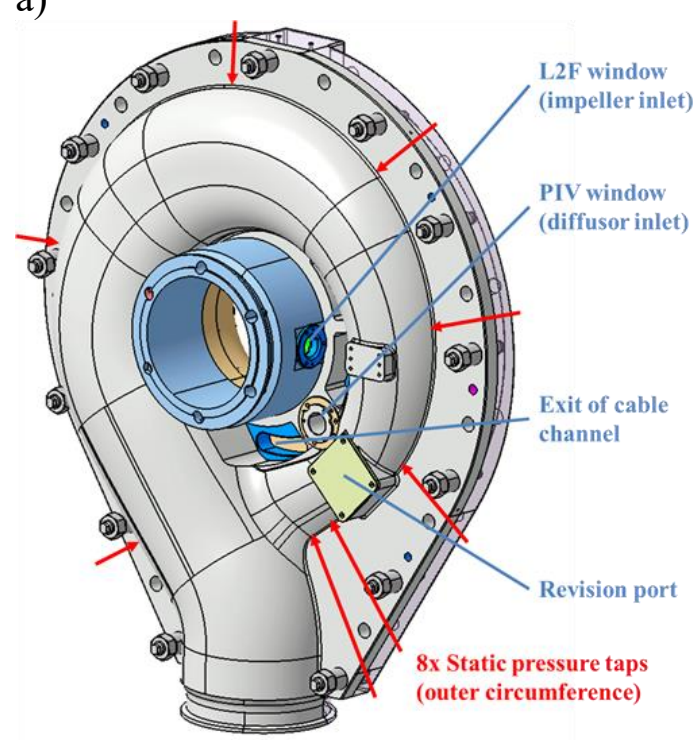

b)

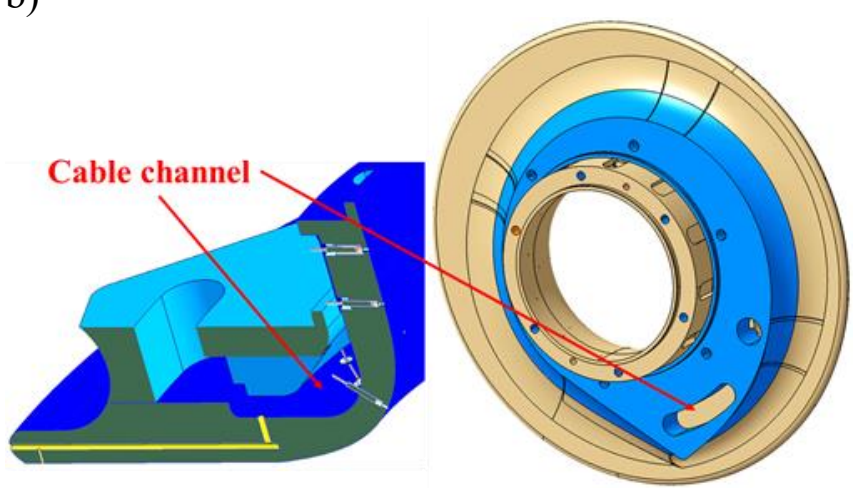

Figure 4: a) Stage design including pressure instrumentation, optical access and revision ports as well as internal cable channel, b) split-design of impeller casing allowing to route wires and tubing needed for static and dynamic pressure sensors, tip clearance monitoring and access port for PIV measurements.

Also visible is a cable channel for the wiring of the internal instrumentation to the exterior as well as the optical access ports for L2F and high-speed PIV. Figure $4 \mathrm{~b}$ provides a $3 \mathrm{D}$ view of the inner casing part covering the impeller and diffuser channel with the lid covering the cable channel marked in 
blue. This part is instrumented with 8 tip clearance sensors and 35 static pressure taps (11 taps in the impeller casing following the meridional flow path contour, 12 taps distributed along the impeller circumference close to the trailing edge, 12 taps in the diffuser casing exit area equally distributed about the circumference). In addition, 19 dynamic pressure sensors (Kulites ${ }^{\circledR}$ ) were integrated in a non-symmetrical distribution at irregular circumferential positions (i.e. not based on the same periodicity when considering characteristic modal structures of unsteady phenomena related to stall and its precursors) forming Kulite arrays at three different meridional positions in the impeller casing. Additional Kulites were placed in the diffuser hub close to the "tongue" of the volute, in proximity of the PIV observation window as well as in the outlet section of the rig.

The inflow conditions directly upstream of the impeller was characterized using L2F measurements. In Figure 5a cross-sectional view of the L2F setup is provided. Due to the restricted access imposed by the volute casing a custom-made mirror device with oblique observation arrangement was necessary to allow optical access for L2F probe beams into the measurement sections.

To gain further insight into the unsteady flow phenomena of the rig time-resolved, high-speed PIV (TR-PIV) was applied in two locations. TR-PIV measurements in the inlet section upstream of the wheel is to characterize the inflow providing time-resolved information at the domain inlet for the accompanying LES and URANS computations. The second TR-PIV measurement domain is located in the diffuser channel as outlined in Figure 6.b. Here the PIV laser light sheet is formed externally before passing a small window integrated into the volute flange. A flat and planar laser window was chosen to avoid beam aberrations or lensing effects, which resulted in a small cavity in the volute flow path.
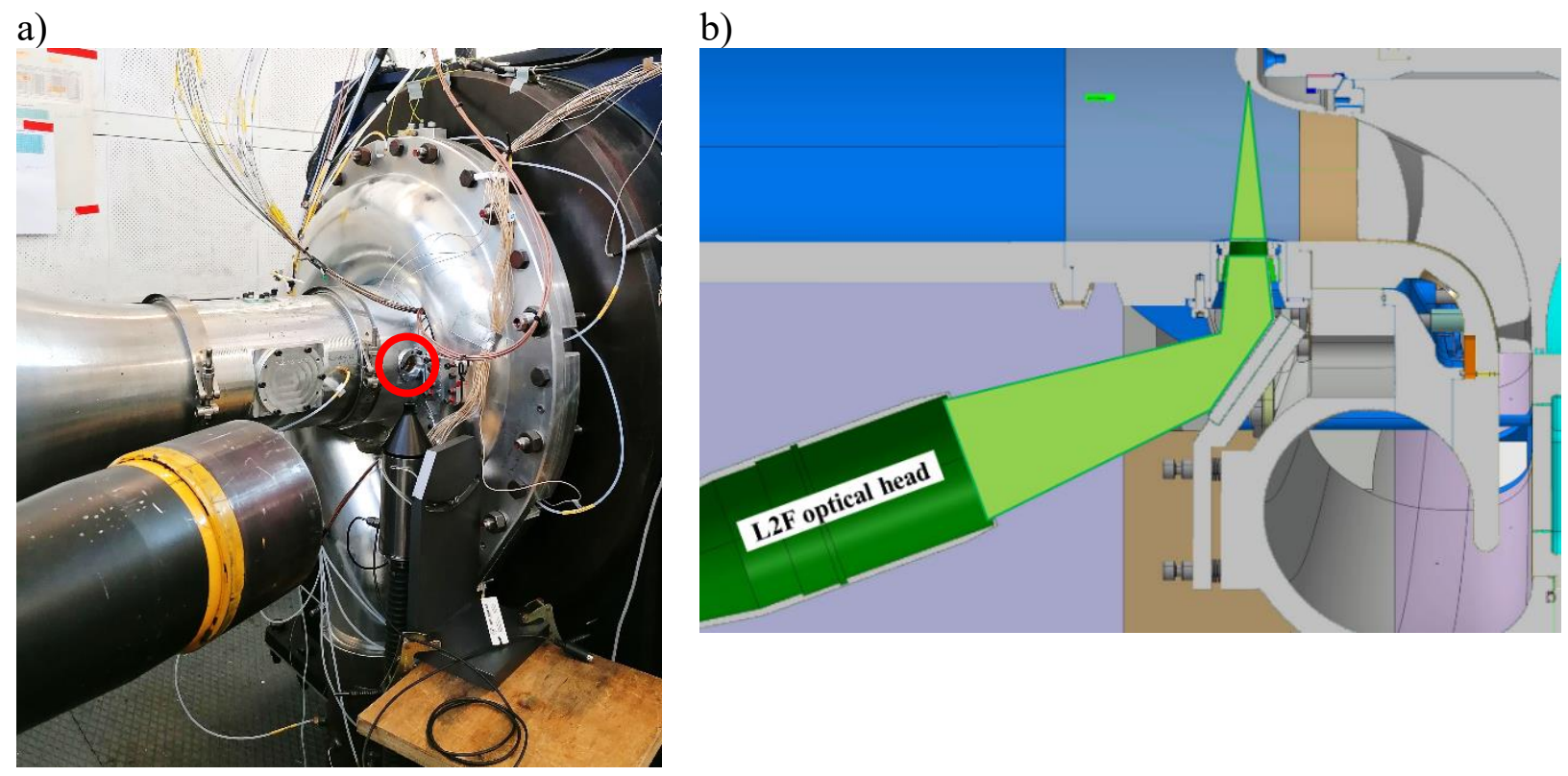

Figure 5: a) Photograph of the L2F setup (red circle marks the optical access for the L2F beams); b) Detail of the optical access for L2F upstream of the impeller leading edge

The TR-PIV measurement approach follows the procedures described by Willert (2015) in which a narrow image domain is captured at high frame rates with large sample counts, typically at high image magnification. In effect, this TR-PIV implementation provides time-records of the velocity profile (e.g. single column of vector data evolving in time) and yields both spectral information and converged statistical quantities. For the present application, the region of interest is illuminated by a pair of diode-pumped, pulsed solid-state lasers (DPSS) whose light is collimated into a $250-400 \mu \mathrm{m}$ thin light sheet. The light scattered by aerosol-based seeding ("oil fog") injected upstream of the rig intake section was imaged by a high-speed camera (Vision Research V1840) at a frame rate of $52 \mathrm{kHz}$, yielding PIV image pairs at $26 \mathrm{kHz}$. Using a $200 \mathrm{~mm}$ macro lens (Nikon Micro-Nikkor 200/4) the image magnification could be set at or above unity, such that spatial scales down to about 
200x200 $\mu \mathrm{m}^{2}$ are captured using PIV sampling of 16x16 pixels. Further details on the above TR-PIV instrumentation can be found in Klinner et al. (2020).

a)

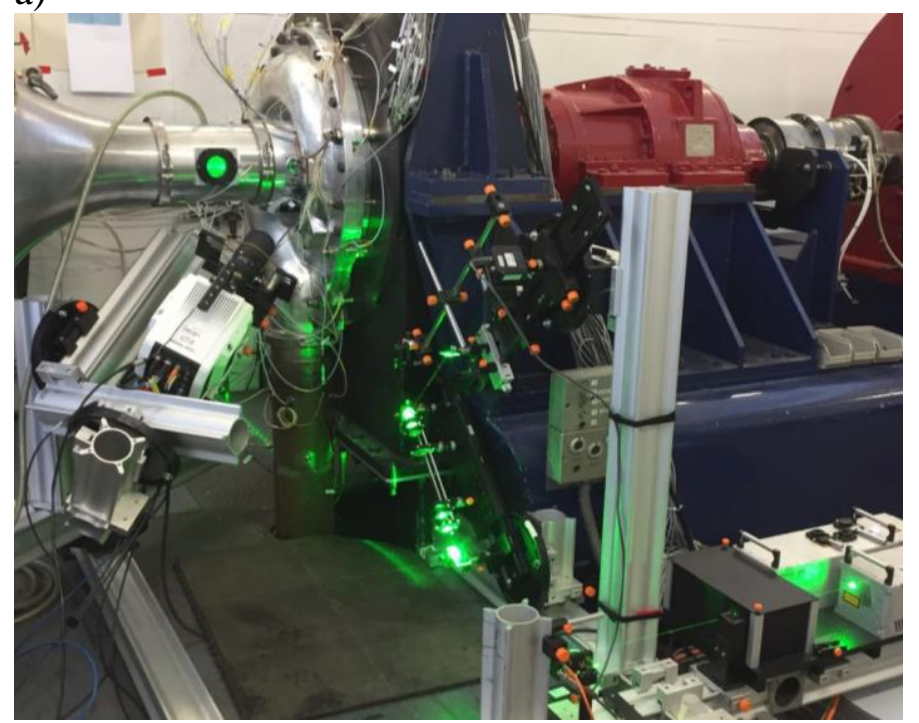

b)

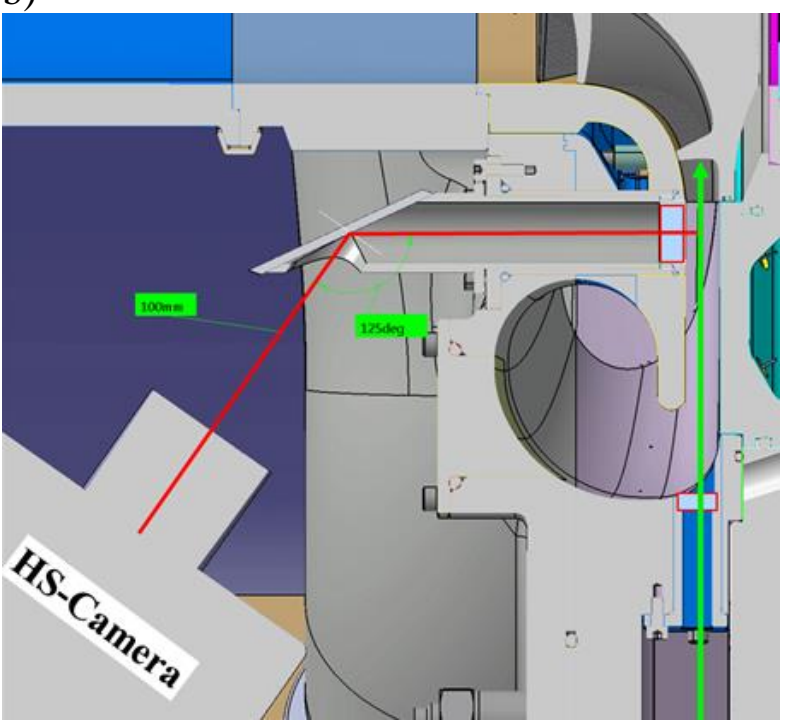

Figure 6: a) Photograph of rig with installed high-speed PIV instrumentation for the investigation of the impeller exit flow, b) corresponding cross-sectional view of the optical access for PIV in the diffuser channel.

For each test point TR-PIV data were recorded as a succession of short $(\sim 0.4 \mathrm{~s})$ bursts each consisting of 10640 PIV samples at a sample rate of $26 \mathrm{kHz}$. To improve statistical convergence up to 16 independent bursts were recorded per rig test point. The TR-PIV measurements were also synchronized with the dynamic pressure measurements allowing for a combined correlation analysis of the transient pressure and velocity information as previously conducted on axial compressor components (see e.g. Pardowitz et al., 2013 \& 2015).

\section{RESULTS OF PERFORMANCE MEASUREMENTS AND SURGE TESTS}

The first part of the test campaign was focused on detailed performance testing of the compressor stage including part-speed and over-speed operation (40\% rpm to $120 \%$ speed) using standard instrumentation and dynamic pressure measurement. As uncontrolled entry into surge should be avoided, the rig was operated very carefully near the numerically predicted stability limit. Online monitoring of the unsteady pressure signals and a fast-opening bypass valve were used to quickly recover from sudden surge events. The performance map, shown in Figure 7, provides six measured speed lines in comparison with the results of the numerical twin of the ROSSINI compressor stage. The agreement between numerical and experimental results is very good, especially regarding the choke-to-peak pressure development. To achieve optimum similarity between numerical and experimental set up the measured tip gap obtained after rig assembly was transferred the computational domain. Re-calculated RANS data with the corrected tip gap are already included in Figure 7 for all speed lines except for the $60 \%$ and $80 \%$ rpm (which are presented with the design tip clearance data) and confirmed the expected improvement, as the experimental and numerical curve show almost identical development. The calculations for the remaining rotating speeds were still in progress while this paper was being written.

In a second step the stability limit and surge behavior of the ROSSINI rig was investigated. A profound knowledge of any precursor of RI or entry into surge is very important for this project, as it is planned to perform TR-PIV measurements at operating conditions with flow instabilities, given that the compressor stage can be stabilized in a safe distance from the surge line.

Online FFT transformations of the Kulite signals were used to identify the beginning of unstable flow conditions and possible entry into RI, which is visible as a low-frequency "hump" below the BPF. 
The throttle valve was gradually closed in small steps while recording Kulite data as long as the rig did not enter into surge. Upon the occurrence of a surge event an automatic fast response bypass valve was rapidly opened allowing for the mass flow to recover and to stabilize the rig allowing it to continue in a safe operating regime.

With the knowledge of the rig surge line from the experiments it turned out that the numerical stability limit of the RANS calculations at high rotational speeds $(100 \%-120 \% \mathrm{rpm})$ reached even lower mass flow rates at higher back pressure (indicated by the grey dashed line in Figure 7). The reason for this is expected to originate from a separation bubble occurring in the volute, that appears to be overestimated in size in the RANS calculations compared to the real rig behavior. Due to this blockage in the flow path of the volute and the related redistribution of the mass flow there might be a stabilizing effect on the impeller passage flow. This hypothesis has to be further investigated as soon as all the measurement data and numerical results (including URANS) have been processed and evaluated. In contrary to the higher speed lines, the numerical stability limit for lower rotational speeds was predicted at much higher mass flows than measured during rig performance tests. The main reason for that was due to convergence problems.

The recorded transient pressure data of the Kulites was revolution-averaged and an FFT was performed subsequent to the measurements. Mean power spectra (MPS) and time-resolved power spectra (waterfall diagrams) were computed and show the time history of different throttle positions during rig operation. Figure 8. a show both the MPS and waterfall diagram of a Kulite sensor located at $95 \%$ meridional length. Here the MPS shows the expected hump with low-frequency "ripples", which is an indication that flow instabilities including RI have already developed in the compressor stage.
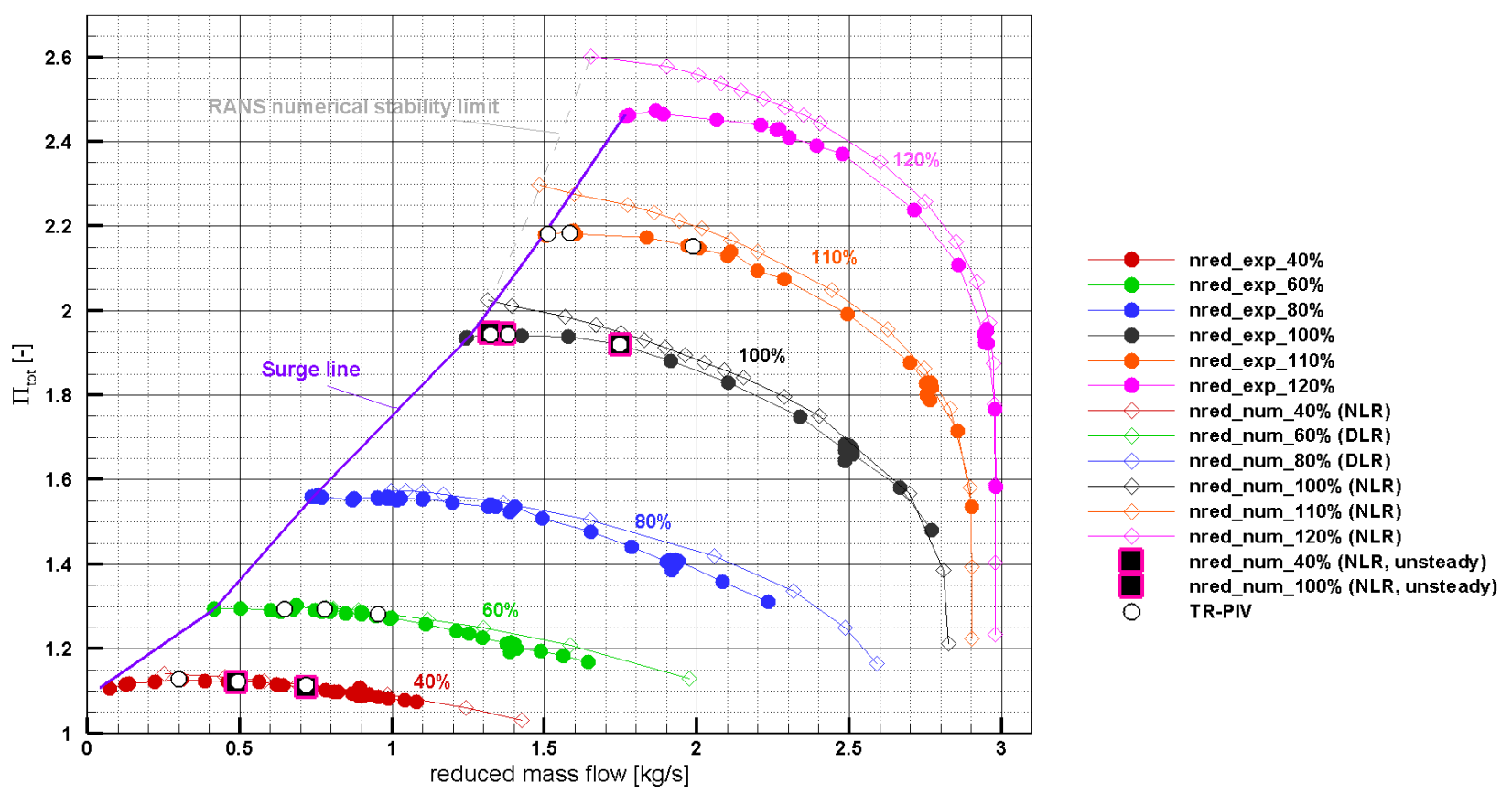

Figure 7: Performance map of the ROSSINI rig comparing RANS and URANS simulations with rig measurement data. Solid circles represent data from experiment, open diamonds from RANS simulation. White circles indicate conditions for TR-PIV measurements.

In the waterfall diagram shown in Figure 8.b the abrupt drop in noise level is an indicator for the fast opening of the bypass valve after a surge event (at $\sim 65 \mathrm{~s}$ on the time axis). As the power spectrum is integrated over the recording time (shown on the right side of Figure 8.b), the variation of the MPS due to the recovered flow situation is not captured. With the knowledge of the exact mass flow and throttle position at surge entry, the TR-PIV measurement points were chosen just before the stage enters into surge, thus enabling an investigation of flow instabilities - or if possible RI - at the last 
stable operating point that can be adjusted without drifting into surge. For these specific operating points URANS calculations have been performed in order to capture the unsteady flow features in the numerical calculations as well. The URANS calculations are still ongoing process while this paper was written. The available results at $40 \%$ and $100 \%$ rotating speed are shown in Figure 7 and are in perfect agreement with the rig measurements.

a)
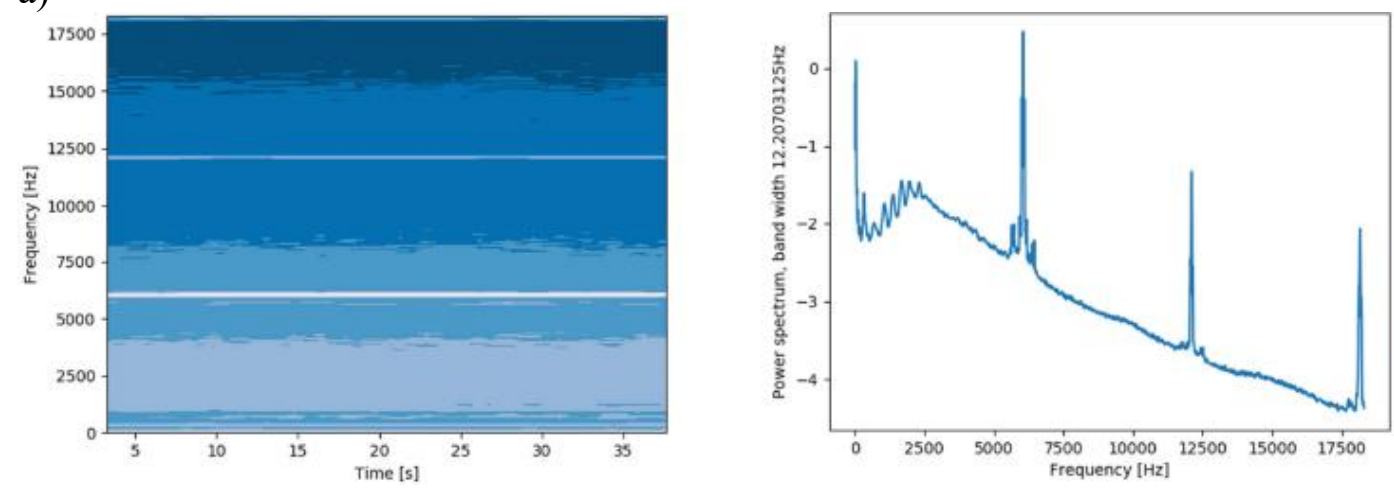

b)
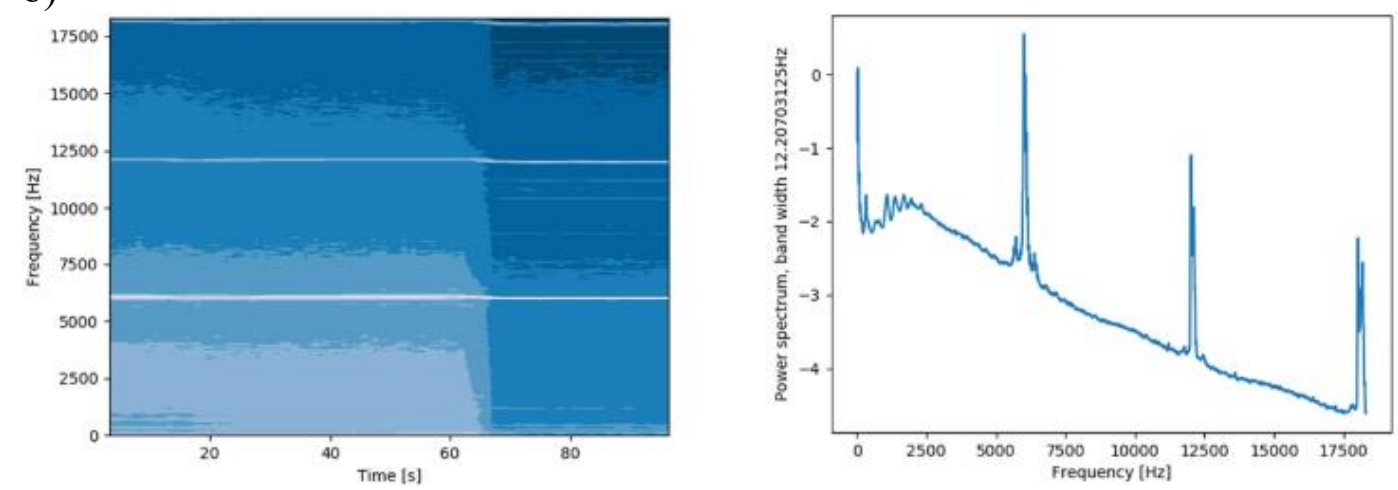

Figure 8: Spectral data of the dynamic pressure probe a) near surge and b) surge event at $100 \%$ rpm showing characteristic "hump" and RI phenomenon (low-frequency "ripples" below BPF).

a)

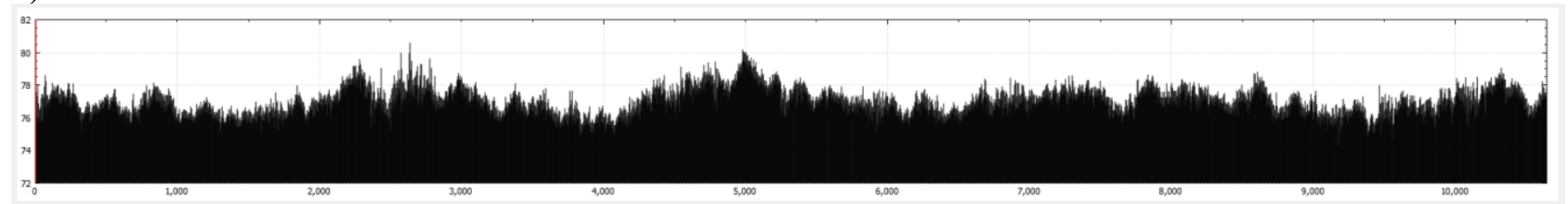

b)

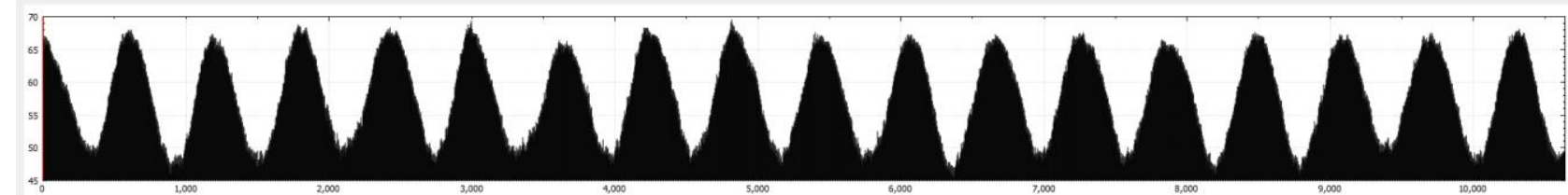

Figure 9: Time record of the streamwise velocity upstream of the impeller at $100 \%$ speed for a "clean" test point (a) and for a RI condition (b). Each record covers a duration of $\sim 0.4 \mathrm{~s}$ (10642 samples). Note that the $y$-axis on a) covers $10 \mathrm{~m} / \mathrm{s} \mathrm{vs} .25 \mathrm{~m} / \mathrm{s}$ for b). 
a)

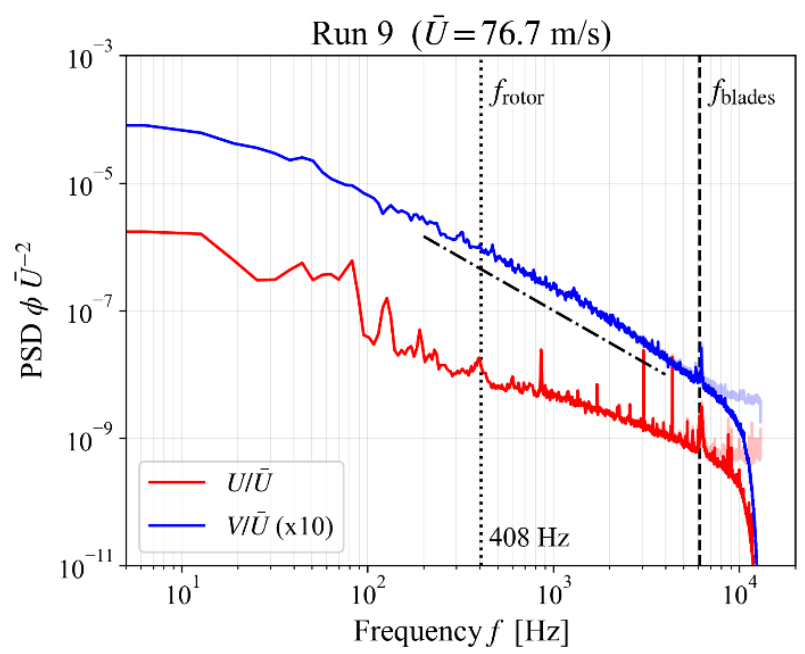

b)

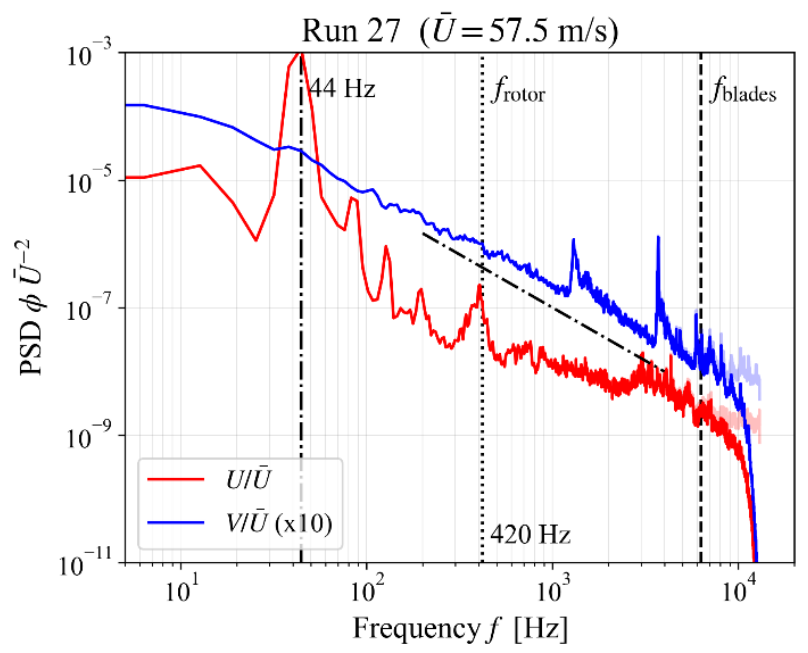

Figure 10: Power spectra of the streamwise (red) and transverse velocity (blue) upstream of the impeller at $\mathbf{1 0 0 \%}$ speed for a "clean" test point (a) and for a RI condition (b). For reference, the dash-dot line indicates the theoretical $-5 / 3$ decay rate of isotropic turbulence. For improved visibility the spectra of the transverse* velocity are scaled by a factor of 10 (* corresponds to the radial velocity component in the light sheet area).

TR-PIV measurements were acquired in the inlet section about $180 \mathrm{~mm}$ upstream of the impeller blade tips at selected conditions at 4 different impeller speeds $(40 \%, 60 \%, 100 \%$ and $110 \%)$. The field of view obtained at the centerline covers $20 \mathrm{~mm}$ in height and $1.8 \mathrm{~mm}$ in streamwise direction. The laser-pulse delay was adjusted to 5.1-6.4 $\mu$ s resulting in rather long mean particle image displacements of $\sim 30$ pixels $(\sim 350 \mu \mathrm{m})$ which improves the SNR of the velocity data. Figure 9 shows representative time records of 10642 PIV recordings $(\sim 0.4 \mathrm{~s})$ of the streamwise velocity components obtained at two different operating conditions. The "clean" condition (Figure 9.a) exhibits a RMS fluctuation of $\sigma_{U} \cong 0.7 \mathrm{~m} / \mathrm{s}$ corresponding to $1 \%$ of the mean. On the other hand, Figure $9 . \mathrm{b}$ shows a strong modulation of the incoming flow $\sigma_{U} \sim 6.4 \mathrm{~m} / \mathrm{s}$ ) with a mean of $U \cong 57.5 \mathrm{~m} / \mathrm{s}$. This is indicative of the presence of a rotating instability condition (RI). Power spectra computed for both velocity components are provided in Figure 10 for the same operating conditions using all 8 available bursts. Noteworthy is the signature of both the blade passing frequency as well as the shaft speed in the spectra. Whereas the "clean" condition exhibits a rather smooth spectrum, the RI condition exhibits a strong frequency peak at $44 \mathrm{~Hz}$ for the axial velocity component, which corresponds to about 1/10 of the impeller speed. The transverse velocity $(V)$ also shows increased dynamics at higher frequencies below the BPF. These characteristics found in the rig measurements are an indication for a single full-span stall cell (acc. to Cumpsty (1989)). Looking at the flow field in the center of the inlet pipe the upstream effect of such a stall cell is expected to result in a modulation of the axial velocity component as visible in Figure 9.b. The exact dynamics of the flow conditions, in particular, in combination with the simultaneously recorded unsteady pressure data is part of an ongoing study and will be addressed in forthcoming publications. TR-PIV measurements in the diffuser have also been performed for the same test conditions but are not described here for reasons of brevity.

\section{CONCLUSIONS}

The current paper described the rig design and instrumentation concept developed for an industrial one-stage centrifugal compressor. The entire rig design and manufacturing process was performed inhouse at DLR Cologne. While testing in laboratory environments provides optimized conditions for the application of advanced flow diagnostics and high-resolution techniques, the most challenging part in the present research work was the integration of the specifically adapted measurement instrumentation in a real turbomachinery component. Results of performance measurements were 
presented, with special focus on the onset of instabilities and surge. This data was fundamental for the choice of relevant test conditions that were further investigated using an array of unsteady pressure probes in combination with TR-PIV, that allow, for instance, a cross-correlation analysis of the signals with respect to one another. The deeper analysis of the extensive data base collected in the course of the experimental campaign, in particular with regard to the precursors of compressor surge, is currently under way and will be covered in upcoming publications.

\section{ACKNOWLEDGEMENTS}

The material presented herein was funded through the Clean Sky 2 Joint Undertaking Project ROSSINI (Radial cOmpreSsor Surge INception Investigation) under the European Union's Horizon 2020 research and innovation program (grant agreement No 717081). The authors gratefully acknowledge the contributions of all project team members, namely Mr. Schindel and the workshop team from DLR, for their effort and valuable contribution during rig design and instrumentation phase.

\section{REFERENCES}

Bousquet Y (2014): Modélisation et analyse des mécanismes impliqués dans l'apparition du pompage d'un étage de compresseur centrifuge, $\mathrm{PhD}$ Thesis, Université de Toulouse, France

Cumpsty, NA (1989) Compressor Aerodynamics, chapter 9, ISBN 0-582-01364-X

Dazin A, Cavazzini G, Pavesi G, Dupont P, Caignaert G, Bois G and Ardizzon G (2009): Rotating Instability in a radial vaneless diffuser, Part. 1: High speed stereoscopic PIV measurements, 8th European Conference on Turbomachinery, Graz, Austria

Dickmann HP, Wimmel TS, Szwedowicz J, Filsinger D and Roduner CH (2006): Unsteady flow in a turbocharger centrifugal compressor: three dimensional fluid dynamics simulation and numerical and experimental analysis of impeller blade vibration. ASME J Turbomach. 128:455-465

Elfert M, Weber A, Wittrock D, Peters A, Voss C and Nicke E (2017): Experimental and Numerical Verification of an Optimization of a Fast Rotating High-Performance Radial Compressor Impeller. ASME J Turbomach. 139 (10) 101007-1. DOI: 10.1115/1.4036357, ISSN 0889-504X

Ferrara G, Ferrari L and Baldassarre L (2004): Rotating stall in a centrifugal compressor Vaneless Diffuser: Experimental Analysis of Geometrical Parameters Influence on Phenomenon Evolution, Int. J of Rot. Machinery 10(6): 433-442

Fisher FB (1988): Application of map width enhancement devices to turbocharger compressor stages. SAE Technical Paper. DOI 10.4271/880794

Guillou E, Gancedo M, Gutmark E and Mohamed A (2012): PIV investigation of the flow induced by a passive surge control method in a radial compressor. Exp Fluids 53:619-635, DOI 10.1007/s00348012-1310-8

Klinner J, Hergt A, Grund S, Willert C (2020): High-Speed PIV of shock boundary layer interactions in the transonic buffet flow of a compressor cascade. Exp. Fluids (accepted, to appear).

Pardowitz B, Tapken U, Sorge R, Thamsen PU and Enghardt L. (2013): Rotating Instability in an Annular Cascade: Detailed Analysis of the Instationary Flow Phenomena. ASME J Turbomach. 136 (6) 061017-1, DOI: 10.1115/1.4025734

Pardowitz B, Peter J, Tapken U, Thamsen PU and Enghardt L (2015): Visualization of Secondary Flow Structures Caused by Rotating Instability: Synchronized Stereo High-Speed PIV and Unsteady Pressure Measurements, 45th AIAA Fluid Dynamics Conference, Dallas, Texas, AIAAAviation 2015, DOI: $10.2514 / 6.2015-2930$

Pavesi G, Dazin A, Cavazzini G, Caignaert G, Bois G and Ardizzon G (2011): Experimental and Numerical Investigation of Unforced unsteadiness in a Vaneless Radial Diffuser, 9th European Conference on turbomachinery - fluid dynamics and thermodynamics, Istanbul, Turkey

Pullan G, Young AM, Day IJ, Greitzer EM and Spakovszky ZS (2012): Origins and structure of spiketype rotating stall. Proceedings of ASME Turbo Expo, GT2012-68707, Copenhagen, Denmark Rosemeier J (2017): Numerical Analysis of a Centrifugal Compressor Including a Vaneless Diffuser and a Volute. 17th ODAS2017, Aussois, France 
Sorokes JM (2003): Rotating stall - an overview of Dresser-Rand experience. Dresser-Rand, Olean, USA

Trébinjac I, Bulot N, Ottavy X, and Buffaz N (2011): Surge inception in a transonic centrifugal compressor stage. Proceedings of ASME Turbo Expo, GT2011-45116, Vancouver, Canada

Weber A, Morsbach C, Kügeler E, Rube C and Wedeking M (2016): Flow analysis of a high flowrate centrifugal compressor stage and comparison with test rig data. Proceedings of ASME Turbo Expo, GT2016-56551

Weichert S and Day IJ (2012): Detailed measurements of spike formation in an axial compressor. Proceedings of ASME Turbo Expo, GT2012-68627, Copenhagen, Denmark

Willert CE (2015): High-speed particle image velocimetry for the efficient measurement of turbulent statistics. Exp Fluids 56:17. DOI 10.1007/s00348-014-1892-4

Yamaguchi S, Yamaguchi HI, Goto S, Nakao H, Nakamura F (2002): The development of effective casing treatment for turbocharger compressors. Proceedings of the 7th IMechE Intern. Conf. Turbochargers and Turbocharging C602/008/2002, London, UK 